\title{
ENDOVASCULAR THERAPY IN BASILAR ARTERY OCCLUSION: IMPACT \\ OF RECANALIZATION, THROMBUS BURDEN AND COLLATERALS
}

The Royal Melbourne Hospital

F. Alemseged ${ }^{1,2}$, E. Van der Hoeven ${ }^{3}$, F. Di Giuliano ${ }^{4}$, D. Shah ${ }^{1}$, N. Yassi ${ }^{1}$, F. Arba ${ }^{5}$,T. Kleinig ${ }^{6}$, S. Bush ${ }^{7}$, R. J. Dowling ${ }^{7}$ MBBS, B. Yan ${ }^{1}$, F. Sallustio ${ }^{2}$, R. Gandini $^{8}$, G. A. Donnan 9 , V. Puetz ${ }^{10}$, M. Diomedi² ${ }^{2}$ M. Parsons ${ }^{1}$, P. J. Mitchell7, S. M. Davis ${ }^{1}$, W. Schonewille ${ }^{11}$, B.C.V. Campbell ${ }^{1}$

"Department of Medicine and Neurology, University of Melbourne, Australia; 2Stroke Unit, University Hospital of "Tor Vergata", Rome, Italy; ' ${ }^{2}$ epartment of Radiology, St. Antonius Hospital, the Netherlands; "Department of Biomedicine and Prevention, University Hospital of "Tor Vergata", Rome, Italy; ${ }^{5}$ Careggi University Hospital, Florence, Italy; ${ }^{6}$ Department of Neurology, Royal Adelaide Hospital, Australia; 'Department of Radiology, Royal Melbourne Hospital, Australia; ${ }^{8}$ Department of Interventional Radiology and Neuroradiology, University of Tor Vergata, Rome, Italy; ${ }^{9}$ The Florey Institute of Neuroscience and Mental Health, Australia; ${ }^{10}$ Department of Neurology and Dresden Neurovascular Center, Dresden University of Technology, Germany; ${ }^{11}$ Department of Neurology, St. Antonius Hospital, the Netherlands

\section{Background}

Whether radiological factors influence the effect of recanalization on outcome in basilar artery occlusion (BAO) is uncertain. ${ }^{1}$ We evaluated the associations between recanalization, thrombus burden, collaterals and outcome in endovascular BAO patients.

\section{Methods}

Clinical and radiological data of endovascular stroke patients with BAO diagnosed on CT angiography at Royal Melbourne Hospital and Royal Adelaide Hospital (Australia), University Hospital of Tor Vergata and Careggi University Hospital (Italy) and from the international BASICS registry ${ }^{2}$ were retrospectively analysed. Two investigators assessed the Basilar Artery on Computed Tomography Angiography (BATMAN) score (Figure 1) ${ }^{3}$, blinded to clinical outcome. Previously validated dichotomy for favourable BATMAN score $(\geq 7)$ was used. Good outcome was defined as modified Rankin Scale $\leq 3$ within 3months; successful reperfusion as $\mathrm{mTICI} 2 \mathrm{~b}-3$ (or TIMI 2-3 in BASICS).

\section{Results}

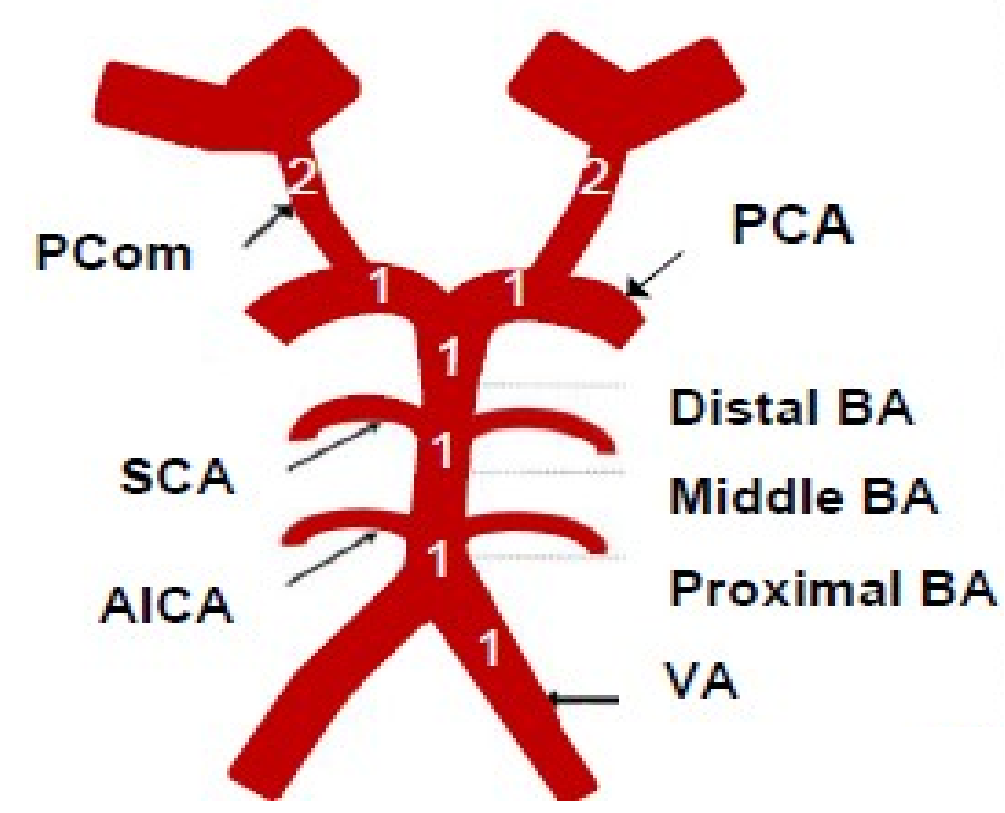

Figure 1. Basilar Artery on ToMography Angiography score. BA basilar artery; VA vertebral artery; PCA posterior cerebral artery; SCA superior cerebellar artery; AICA antero-inferior cerebellar artery; PCOM posterior communicating artery.

We included 170 BAO patients treated with intra-arterial urokinase and/or mechanical thrombectomy (122 with only mechanical thrombectomy): mean(SD) age 65(13), median NIHSS 22(IQR 12-30), 36.5\% treated beyond 6 hours. Recanalization was achieved in $79 \%$ patients and was associated with good outcome $(p=0.003)$.

\begin{tabular}{|c|c|c|c|c|c|c|}
\hline \multicolumn{7}{|c|}{$\mathrm{N}=170$ endovascular BAO patients } \\
\hline & \multicolumn{3}{|c|}{$\begin{array}{c}\text { Favorable } \\
\text { BATMAN score (7-10) } \\
n=61\end{array}$} & \multicolumn{3}{|c|}{$\begin{array}{c}\text { Unfavorable } \\
\text { BATMAN score (0-6) } \\
n=109\end{array}$} \\
\hline & OR & $95 \% \mathrm{Cl}$ & $\mathbf{P}$ & OR & $95 \% \mathrm{Cl}$ & p \\
\hline $\begin{array}{l}\text { Successful } \\
\text { reperfusion }\end{array}$ & 11 & $1.2-98$ & 0.03 & 3.7 & $0.7-20$ & 0.1 \\
\hline Age & 0.9 & $0.8-0.9$ & 0.01 & 0.9 & $0.9-1.1$ & 0.2 \\
\hline NIHSS & 0.9 & $0.8-0.9$ & 0.003 & 0.9 & $0.9-1.1$ & 0.3 \\
\hline OTT $<6$ hours & 3.5 & $0.7-18$ & 0.1 & 7.2 & $1.5-35$ & 0.01 \\
\hline \multicolumn{7}{|c|}{$\begin{array}{l}\text { Table 1. In logistic regression (adjusted for age, NIHSS and time to treatment), } \\
\text { recanalization remained associated with good outcome (OR } 11,95 \% \mathrm{Cl} 1.2-98 \text {, } \\
\mathrm{p}=0.03 \text { ) in patients with favourable BATMAN score. Treatment within } 6 \text { hours } \\
\text { was associated with good outcome only in patients with unfavourable BATMAN } \\
\text { score (adjusted OR } 7.2,95 \% \mathrm{Cl} 1.5-35, \mathrm{p}=0.01 \text { ). OTT onset time to treatment; } \\
\text { NIHSS National Institutes of Health Stroke Scale; BATMAN Basilar Artery } \\
\text { ToMogranhv ANGiogranhv score }\end{array}$} \\
\hline
\end{tabular}

\section{Conclusions}

Recanalization is associated with good outcome in BAO patients with good collaterals and less extensive occlusion, even in delayed time window.

Singer OC, Berkefeld J, Nolte CH, Bohner G, Haring HP, Trenkler J, Gröschel K, Müller-Forell W, Niederkorn K, Deutschmann H, Neumann-Haefelin T, Hohmann C, Bussmeyer M, Mpotsaris A, Stoll A, Bormann A, Brenck J, Schlamann MU, Jander S, Turowski B, Singer OC, Berkefeld J, Nolte CH, Bohner G, Haring HP, Trenkler J, Gröschel K, Müller-Forell W, Niederkorn K, Deutschmann H, Neumann-Haefelin T, Hohmann C, Bussmeyer M, Mpots
Petzold GC, Urbach H, Liebeskind DS; ENDOSTROKE Study Group. Mechanical recanalization in basilar artery occlusion: the ENDOSTROKE study. Ann Neurol. 2015 Mar;77(3):415-24.

Schonewille WJ, et al; BASICS study group. Treatment and outcomes of acute basilar artery occlusion in the Basilar Artery International Cooperation Study (BASICS): a prospective registry study. Lancet Neurol. 2009 Aug;8(8):724-30.

Alemseged F, Shah DG, Diomedi M, Sallustio F, Bivard A, Sharma G, Mitchell PJ, Dowling RJ, Bush S, Yan B, Caltagirone C, Floris R, Parsons MW, Levi CR, Davis SM, Campbell BC. The Basilar Artery on Computed Tomography Angiography Prognostic Score for Basilar Artery Occlusion. Stroke. 2017 Mar;48(3):631-637. 